\title{
The 3-min Test Does not Provide a Valid Measure of Critical Power Using the SRM Isokinetic Mode
}

\author{
Authors

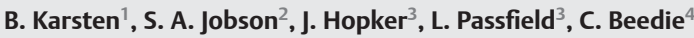 \\ Affiliations \\ ${ }^{1}$ Life and Sports Science, University of Greenwich, Chatham Maritime, United Kingdom \\ ${ }^{2}$ Department of Sports Studies, University of Winchester, United Kingdom \\ ${ }^{3}$ Centre for Sports Studies, University of Kent, Chatham Maritime, United Kingdom \\ ${ }^{4}$ Department of Sport and Exercise Science, Aberystwyth University, Aberysthwyth, United Kingdom
}

Key words
critical intensity
exercise testing
anaerobic work capacity
reliability
validity

accepted after revision May 14, 2013

Bibliography DOI http://dx.doi.org/ 10.1055/s-0033-1349093 Published online: November 10, 2013 Int J Sports Med 2014; 35 : 304-309 (c) Georg Thieme Verlag KG Stuttgart · New York ISSN 0172-4622

Correspondence

Bettina Karsten

Life and Sports Science

University of Greenwich

Central Avenue

ME4 4TB Chatham Maritime

United Kingdom

Tel.: $+44 / 208 / 3317927$

Fax: +44/208/3319805

kb20@gre.ac.uk

\section{Abstract}

$\nabla$

Recent datas suggest that the mean power over the final $30 \mathrm{~s}$ of a 3-min all-out test is equivalent to Critical Power (CP) using the linear ergometer mode. The purpose of the present study was to identify whether this is also true using an "isokinetic mode”. 13 cyclists performed: 1) a ramp test; 2) three 3-min all-out trials to establish End Power (EP) and work done above EP (WEP); and 3 ) 3 constant work rate trials to determine $\mathrm{CP}$ and the work done above $\mathrm{CP}\left(W^{\prime}\right)$ using the work-time $\left(=\mathrm{CP} 1 / W^{\prime} 1\right)$ and $1 /$ time $\left(=\mathrm{CP} 2 / W^{\prime} 2\right)$

\section{Introduction}

Critical power (CP), defined as the highest sustainable rate of aerobic metabolism [17], demarcates the heavy and the severe exercise intensity domains [16,21,29], and is conceived as an intensity that can be maintained over time without eliciting $\mathrm{VO}_{2 \max }$ [21]. The measurement of $\mathrm{CP}$ and its related finite quantity of 'anaerobic' energy $\left(W^{\prime}\right)$, which is a marker of the build-up of fatigue-inducing metabolites to a tolerable limit, has received considerable recent research attention $[13,25,29]$.

$\mathrm{CP}$ is traditionally estimated via repeated, multiday, exhaustive exercise tests. This arguably reduces its practical utility [18]. Several authors have investigated the validity of single 'all-out' tests to determine $\mathrm{CP}[8,14,15,29]$. Given that any exercise bout performed above $\mathrm{CP}$ should lead to the gradual expenditure of $W$, a sufficiently long all-out exercise bout should lead to the attainment of CP [30].

Based on evidence that $W^{\prime}$ depletion takes $<60 \mathrm{~s}$ $[2,19]$, Brickley et al. [8] hypothesized that power output at the end of a 90-s all-out test would be equivalent to $\mathrm{CP}$. However, the final power output reported by Brickley et al. was signifi- models. Coefficient of variation in EP was $4.45 \%$ between trials 1 and 2, and $4.29 \%$ between trials 2 and 3. Limits of Agreement for trials 1-2 and trials $2-3$ were $-2 \pm 38 \mathrm{~W}$. Significant differences were observed between EP and CP1 $(+37 \mathrm{~W}$, $\mathrm{P}<0.001)$, between WEP and $W^{\prime} 1(-6.2 \mathrm{~kJ}$, $\mathrm{P}=0.001)$, between EP and $\mathrm{CP} 2(+31 \mathrm{~W}, \mathrm{P}<0.001)$ and between WEP and $W^{\prime} 2(-4.2 \mathrm{~kJ}, \mathrm{P}=0.006)$. Average SEE values for EP-CP1 and EP-CP2 of $7.1 \%$ and $6.6 \%$ respectively were identified. Data suggest that using an isokinetic mode 3-min allout test, while yielding a reliable measure of EP, does not provide a valid measure of $\mathrm{CP}$.

cantly higher than CP. Subsequently, Vanhatalo et al. [30] investigated the efficacy of a 3-min allout cycling test and reported that mean power output for the final 30s (End Power or EP) matched CP. Burnley et al. [9] further demonstrated the reliability of EP using three 3-min tests. These results led Poole [29] to state that "the 3 min test promises to herald a new era for experimental exercise physiology". In fact, EP has already been used successfully in a range of settings $[12,26,32]$.

The work of Vanhatalo and colleagues [30] suggests that the power profile of all-out cycle exercise has a fundamental physiological basis. If this is true, similar levels of agreement between allout end-test muscle performance and CP should be observed irrespective of the mode of measurement [25]. However until very recently, published studies of the 3-min test in cycling were conducted using the linear mode setting of the Lode Excalibur Sport ergometer [2,16,30-32]. The degree to which the high level of agreement between parameters reported by Vanhatalo et al. [30] is mechanistic or coincidental has not been independently established. Recently, Bergstrom et al. [4] performed the 3-min test using a Quinton ergometer, also using the linear mode, as well 
as with the Monark ergometer with $3.5 \%$ and $4.5 \%$ of body weight as the set resistance. No agreement between estimates of EP or work done above EP (WEP) values using the Quinton and Monark ergometer were observed.

The aim of the present study was to investigate whether EP estimated using the SRM isokinetic mode would provide a reliable estimate of $\mathrm{CP}$.

\section{Methods}

$\nabla$

\section{Subjects}

12 males and 1 female subject (mean \pm SD: age $33 \pm 7$ year, body mass $78 \pm 14 \mathrm{~kg}$, height $1.79 \pm 0.09 \mathrm{~m}$, Maximal Aerobic Power (MAP) $345 \pm 54 \mathrm{~W}, \dot{\mathrm{V}} \mathrm{O}_{2 \max } 5.18 \pm 0.87 \mathrm{~L} \cdot \mathrm{min}^{-1}$ ) participated in this study. All volunteers were competitive road cyclists with a minimum of 2 years' experience. Subjects refrained from heavy exercise in the $24 \mathrm{~h}$ prior to all tests and from food intake in the $3 \mathrm{~h}$ prior to all tests. The study was conducted in accordance with the ethical standards of the International Journal of Sports Medicine [20] and approved by the University Ethics Committee of the host institution. Prior to providing written informed consent and participation, cyclists were fully informed of the nature and risks of the study.

Exercise testing was conducted on an electronically braked SRM cycle ergometer (Schober Rad Messtechnik, Jülich, Germany). Subjects visited the laboratory 7 times. During visit 1, subjects completed an incremental test to determine $\dot{\mathrm{VO}}_{2 \max }$ and MAP, as well as a 3-min all-out test for familiarization. In visits 2-7 subjects completed 3 constant work rate trials and three 3 -min trials randomly assigned. A standard warm-up of 5-min at $100 \mathrm{~W}$ followed by 5 -min passive rest and 3-min of unloaded cycling [9] was used prior to each trial. During tests the investigator provided consistent and strong verbal encouragement. A post-test blood lactate concentration of $\geq 8 \mathrm{mmol} \cdot 1^{-1}$ or heart rate (HR) within 10 beats of age-predicted HR maximum was taken as an indicator for attainment of $\dot{\mathrm{VO}}_{2 \text { max }}$ and accepted as a successful test [6]. All visits were separated by a minimum of $24 \mathrm{~h}$ and were completed within a maximum period of 21 days. Each subject completed each of their 7 tests at the same time of day.

\section{Protocol}

\section{Maximal oxygen uptake test protocol}

The incremental $\mathrm{VO}_{2 \max }$ test was initiated at a work rate of $150 \mathrm{~W}$. Thereafter, work rate increased by $20 \mathrm{~W} \cdot \mathrm{min}^{-1}$. Subjects were instructed to maintain their preferred cadence throughout the trial. The trials were terminated when cadence fell by more than $10 \mathrm{rpm}$ for more than $10 \mathrm{~s}$. Pulmonary gas exchange was measured breath-by-breath. Subjects wore a facemask (Hand Rudolph, MO) and breathed through a mouthpiece and impeller turbine assembly. Before each test, the gas analyser (MetaMax 3B, Cortex Biophysik, Leipzig, Germany) was calibrated according to the manufacturer's guidelines. $\mathrm{VO}_{2 \max }$ was recorded as the highest mean oxygen consumption over a 30 -s period, while MAP was recorded as the mean power output during this same period.

\section{Critical Power cycling tests}

$\mathrm{CP}$ was estimated from 3 constant work rate tests at power equivalent to $80 \%, 100 \%$ and $105 \%$ MAP. Each trial was estimated to yield times to exhaustion between 2-15 min [21]. Subjects were instructed to sustain the power output at their preferred cadence for as long as possible. Tests were terminated when cadence fell by more than $10 \mathrm{rpm}^{-1}$ for more than $5 \mathrm{~s}$ [30]. Test durations were recorded to the nearest $0.5 \mathrm{~s}$. Blood lactate was sampled at rest before the test and immediately after its completion and analysed using a Biosen C_line (EKF Diagnostic, Barleben, Germany). Consistent with Vanhatalo [30], linear regression was used to provide an estimate of $\mathrm{CP}$ and $W^{\prime}$ using the work-time $\left(\mathrm{W}=\mathrm{CP} t+W^{\prime}\right.$; equation 1$)$ and the power ${ }^{-1} /$ time $^{\prime}$ $\left(\mathrm{P}=W^{\prime}(1 / t)+\mathrm{CP}\right.$; equation 2$)$ model. Estimates using equation 1 or 2 were consequently termed $\mathrm{CP} 1$ and $\mathrm{CP} 2$.

\section{3-min all-out cycling tests}

During the 3-min test the resistance on the pedals was provided by the SRM ergometer in isokinetic mode, and cadence was therefore maintained at the subjects' preferred level throughout. Subjects were instructed to attain peak power as quickly as possible from the start, and to maintain maximum power throughout the $3 \mathrm{~min}$. To facilitate this, during the final $10 \mathrm{~s}$ of the standard warm-up subjects increased cadence by 10-20 rev $\cdot \mathrm{min}^{-1}$ above preferred cadence. Consistent with Vanhatalo et al. [30] subjects were not informed of elapsed time. End Power (EP) was calculated as the mean power output over the final 30 s of the test. Work done above EP (WEP) was calculated as the power-time integral above EP. Blood lactate was sampled and analysed at rest before the test and immediately after its completion.

\section{Statistical analysis}

Data were examined using the Shapiro-Wilks' normality test. Coefficients of variation $(\mathrm{CoV})$ were derived from log-transformed data [23]. 95\% confidence intervals were calculated for each CoV. Repeated measures ANOVA was used to test for significant differences between 3-min trial 1 and trial 2 and between trial 2 and trial 3. Consistent with Vanhatalo et al. [30], agreement between EP and CP1, WEP and $W^{\prime} 1$, EP and CP2 and WEP and $W^{\prime} 2$ for both models was assessed using a paired-samples $t$-test and limits of agreement (LoA) $[1,7]$. Relationships were assessed using Pearson product moment correlation coefficients. Additionally, linear regression was used to calculate values for Standard Error of Estimates (SEE) to estimate error associated with predicting EP and WEP values. Statistical significance was accepted at $P<0.05$. Results are reported as mean \pm SD unless otherwise stated.

\section{Results}

\section{$\nabla$}

ANOVA indicated no significant differences in EP between pairs of trials, $F(2,26)=0.83, P>0.05$. CoV for EP was $4.45 \%$ between trials 1 and 2 and $4.29 \%$ between trials 2 and 3. Bland-Altman plots of the test-retest data are presented in $\bullet$ Fig. 1. The EP 95\% LoA for trials $1-2$ was $-2 \pm 37 \mathrm{~W}(0.99 * / \div 1.14$ as a ratio $)$ and for trials $2-3$ it was $-4 \pm 35 \mathrm{~W}\left(0.98^{*} / \div 1.13\right.$ as a ratio). The intraclass correlation coefficient for EP values was 0.97 (95\% CI $=0.92-$ 0.99).

$\mathrm{CP}$ and mean EP were normally distributed. Statistically significant differences were observed between EP and CP1 (EP=290 $\pm 41 \mathrm{~W}$ vs. $\mathrm{CP} 1=253 \pm 41 \mathrm{~W}, t(12)=-6.16, P<0.001)$ and between EP and CP2 $(E P=290 \pm 41 W$ vs. $C P 2=259 \pm 38 W, t(12)=-4.645, P<0.001)$. The SD of the differences for CP1 vs. EP was $19 \mathrm{~W}$, providing $95 \%$ LoA of $25 \pm 48 \mathrm{~W}$ ( $\odot$ Fig. 2 c; $0.87^{*} / \div 1.16$ as a ratio) and for CP2 vs. EP the SD of the difference was $18 \mathrm{~W}$, providing $95 \%$ LoA between 
$20 \pm 41 \mathrm{~W}$ ( $\odot$ Fig. $2 \mathrm{~d} ; 0.89^{*} / \div 1.14$ as a ratio). The correlation coefficient for EP and CP1 was $r=0.89, P \leq 0.001$ ( $\odot$ Fig. 2a) and for EP and CP2 r=0.90, $P \leq 0.001$ ( $\bullet$ Fig. 2b). Mean $r^{2}$ values for equation 1 were $0.99 \pm 0.01$ (SEE $2.94 \pm 2.23$ ) and for equation 2 $0.94 \pm 0.06$ (SEE 11.96 \pm 6.55 ). The SEE value for the linear relationship between CP1 and EP was 19.49W, CL (14.49-30.22) with an average error prediction of $7.7 \%$ and for CP2 and EP it was $17.10 \mathrm{~W}, \mathrm{CL}(12.79-26.52)$ with an average error prediction of $6.6 \%$.

Significant differences were observed between WEP and $W^{\prime} 1$ $\left(\mathrm{WEP}=12.5 \pm 4.3 \mathrm{~kJ}\right.$ vs. $\left.W^{\prime} 1=18.6 \pm 4.8 \mathrm{~kJ}, t(12)=-4.65, P=0.001\right)$ and between WEP and $W^{\prime} 2\left(W^{\prime}=16.6 \pm 4.8 \mathrm{~kJ}, t(12)=-3.3\right.$, $P=0.006)$. The SD of the differences was $4.78 \mathrm{~kJ}$ for $W^{\prime} 1 \mathrm{vs}$. WEP, providing $95 \%$ LoA of $3.27 \pm 9.06 \mathrm{~J}$ ( $\bullet$ Fig. 3c; $0.64 * / \div 1.96$ as a ratio) and for $W^{\prime} 2$ vs. WEP the SD of the differences
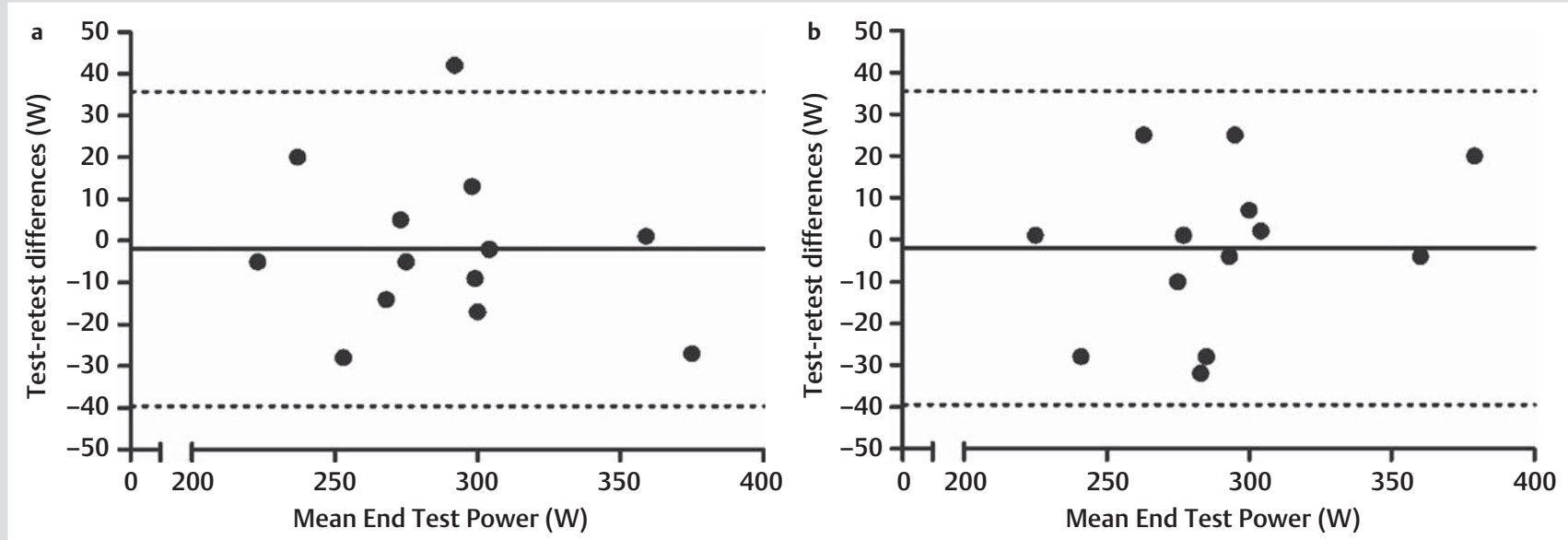

Fig. 1 Bland-Altman plots of the End Power test-re-test differences between trials 1 and 2 a and trials 2 and $3 \mathbf{b}$. The solid horizontal lines represent mean bias, while the dashed lines represent the $95 \%$ LoA.
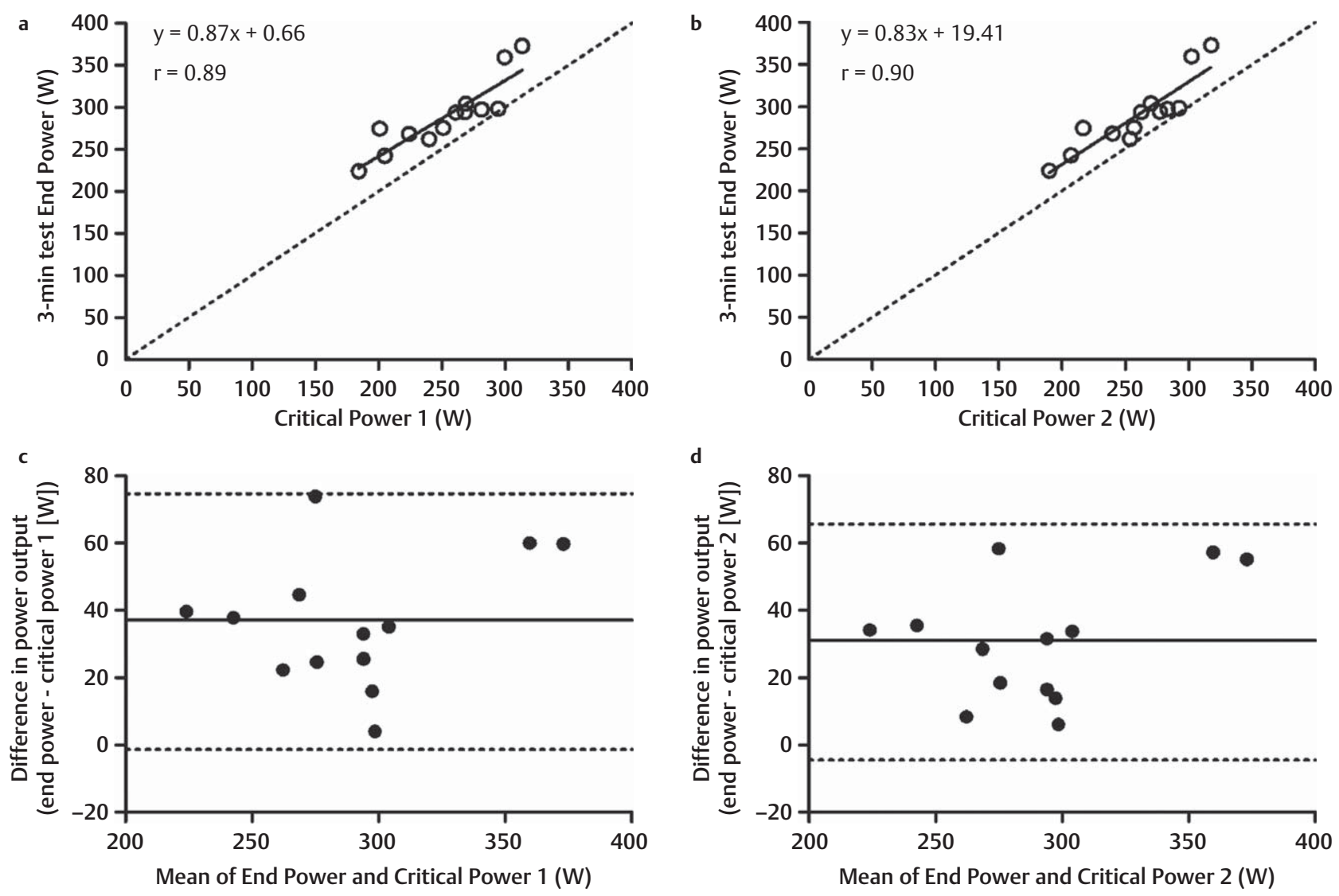

Fig. 2 Bland-Altman plots of the relationship (panel $\mathbf{a}$ and $\mathbf{b}$ ) and limits of agreement (panel $\mathbf{c}$ and $\mathbf{d}$ ) between End Power (W) and CP1 (Watt), and between End Power (W) and CP2 (Watt). In panel $\mathbf{c}$ and $\mathbf{d}$ the solid horizontal line represents the mean difference between End Power and Critical Power 1 and 2 , and the dashed lines represent $95 \%$ LoA. 

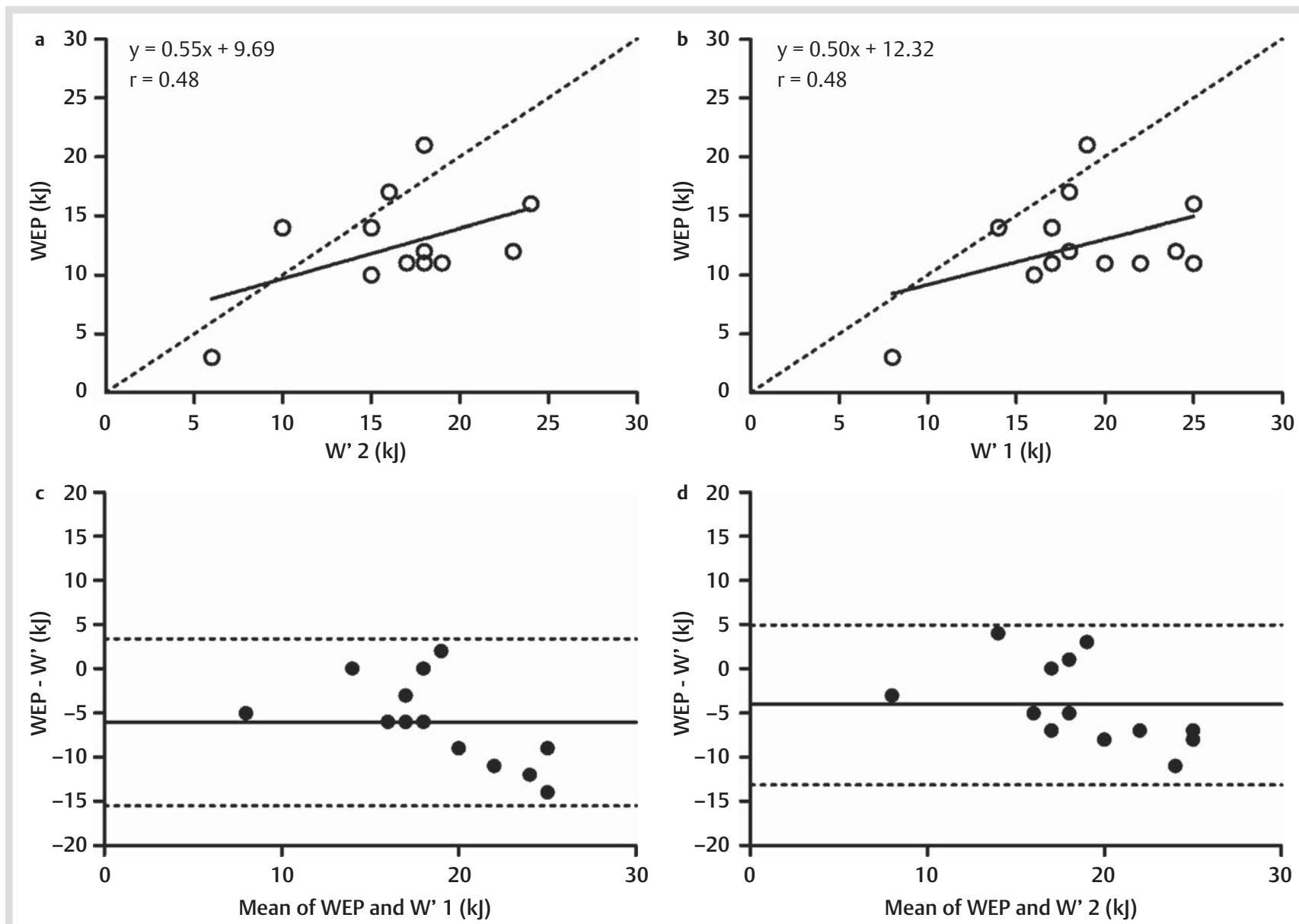

Fig. 3 Bland-Altman plots of the relation (panel $\mathbf{a}$ and $\mathbf{b}$ ) and limits of agreement (panel $\mathbf{c}$ and $\mathbf{d}$ ) between WEP (kJ) and $W^{\prime} 1(\mathrm{~kJ})$ and between WEP ( $\mathrm{kJ}$ ) and $W^{\prime} 2(\mathrm{~kJ})$. In panel $\mathbf{c}$ and $\mathbf{d}$ the solid horizontal line represents the mean difference between End Power and CP 1 and 2 , and the dashed lines represent $95 \%$ LoA.

was $4.53 \mathrm{~kJ}$, providing $95 \%$ LoA of $1.43 \pm 6.90 \mathrm{~kJ}$ ( $\odot$ Fig. 3d; $0.73 * / \div 1.93$ as a ratio). The correlation coefficient for WEP and $W^{\prime} 1$ was $r=0.43, P=0.14$ and for WEP and $W^{\prime} 2 r=0.48, P=0.10$ ( $\bullet$ Fig. 3a, b). The SEE value for the linear relationship between $W^{\prime} 1$ and WEP resulted in $4.5 \mathrm{~kJ}, \mathrm{CL}$ (3.37-6.98) with an average error prediction of $24.2 \%$ and for $W^{\prime} 2$ and WEP it was $4.37 \mathrm{~kJ}, \mathrm{CL}$ (3.27-6.78) with an average prediction error of $26.3 \%$.

\section{Discussion}

$\nabla$

The results presented above suggest that a 3-min all-out cycling test using the SRM isokinetic mode does not provide a valid measure of CP. Specifically, the mean power output during the final $30 \mathrm{~s}$ of the 3-min all-out test appears to be significantly higher than estimates of $\mathrm{CP}$ derived from both work-time and power $^{-1} /$ time models. The 3 -min test also appears to underestimate the 'anaerobic' parameter of the $\mathrm{CP}$ model (i.e., $W^{\prime}$ ). The results presented above also suggest that the 3-min all-out test is a reliable measure of EP when studying a trained athletic population.

A $5 \%$ coefficient of variation (CV) has been cited as an acceptable upper limit in sports science reliability studies [23]. Given that the $\mathrm{CV}$ values observed were below this boundary of $5 \%$, the EP from a 3-min all-out cycling test can be considered to be reliable. In fact, Burnley et al. [9] suggested that EP is a reproducible measure when reporting a coefficient of variation (typical error as a percentage of the mean) only a little lower than that reported here (3\% vs. 4.9\%). Johnson et al. [24] reported a CV of $6.7 \%$ for the 3-min all-out EP results, and even given this accepted the test as reliable. However, caution should be taken as such a level of variation is unlikely to be acceptable when evaluating the relatively small training-induced changes seen in welltrained athletes [27]. Such a conclusion is supported by limits of agreement analyses which suggest that, with an approximate 95\% probability, the differences between the test and retest of EP in a well-trained cyclist will lie between $-40 \mathrm{~W}$ and $+36 \mathrm{~W}$. Assuming that the bias is negligible, ratio limits of agreement suggest that, between any 2 tests, EP will differ by as much as $14 \%$ in a positive or negative direction. Using a magnitude-based analysis, Paton and Hopkins [28] identified that a change of $1.7 \%$ in performance impacts the chances of an elite road time trial cyclist winning an event. With an average SEE value for EP-CP 1 and EP-CP 2 of $7.7 \%$ and $6.6 \%$, respectively, the discrepancy between the 2 measurement methods in the present study would therefore result in substantial performance differences. In a heterogeneous group of cyclists, runners and fitness trained subjects, Vanhatalo et al. [30] reported no differences between EP $(287 \pm 55 W)$ and CP $(287 \pm 56 W)$. In contrast, in the present study EP was significantly higher than CP1 and CP2 (37 W and $31 \mathrm{~W}$ respectively). Several factors might explain this lack of agreement. First, it is possible that the use of 3 constant work 


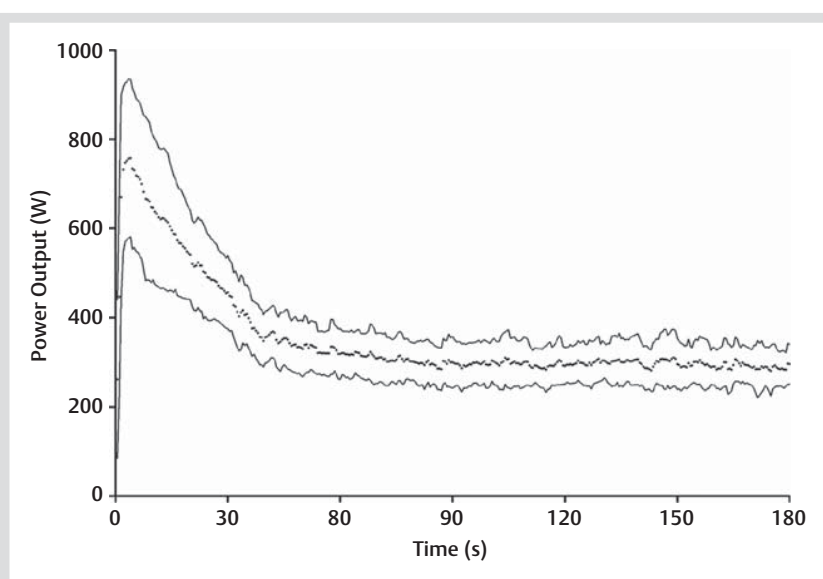

Fig. 4 Group mean power profile of the SRM isokinetic 3-min all-out cycle test. Solid lines represent the standard deviation.

rate trials resulted in an inaccurate estimate of $\mathrm{CP}$ and $W^{\prime}$. Vanhatalo et al. [30] used 5 trials, while research seeking to model the power-exhaustion time relationship commonly uses 4 or more trials $[3,10]$. However, several recent investigations have used 3 tests for CP and $W$ ' estimation $[2,15]$. According to Hill [22] the decision as to the number of trials used depends on the fitness level of subjects as well as their familiarity with all-out exercise. Subjects in the present study were accustomed to allout exercise, a fact which arguably justified the use of 3 trials in line with Hill's proposal. Strong correlation and low SEE values observed for each subject and model used lend further support to this decision (mean $\mathrm{r}^{2}$ values for equation 1 was $0.99 \pm 0.01$ / SEE $2.94 \pm 2.23$ and for equation 2 it was $0.94 \pm 0.06 /$ SEE $11.96 \pm 6.55)$. Secondly, as pulmonary gases were not recorded during the 3-min all-out tests, it might be suggested that we did not meet all 3 conditions outlined by Jones et al. [25] for the attainment of a successful 3-min test (i.e., that subjects did not reach sufficiently high intensity). However, the post-test lactate concentrations $\left(12.3 \pm 3.8 \mathrm{mmol} \cdot \mathrm{L}^{-1}\right)$ were higher than those reported by Vanhatalo et al. [30] $\left(10.2 \pm 2.2 \mathrm{mmol} \cdot \mathrm{L}^{-1}\right)$. Given that all subjects also reached values within 10 beats per minute of their age-predicted maximal heart rates, we are confident that subjects did perform at an appropriate intensity. Furthermore, the group mean power profile suggests both the very high intensities achieved during the first 60 s of the all-out trials and the subsequent plateau, both of which are vital to the proposed efficacy of the 3-min test ( $\bullet$ Fig. 4).

It is also possible that the discrepancy between the present results and those of Vanhatalo et al. [30] relate to the use of different ergometers. The isokinetic mode of the SRM allows the cyclist to maintain a fixed cadence while the resistance adapts to any change in pedal force. In contrast, in the linear mode of the Lode the applied resistance is cadence-dependent, and in the early stages of the 3-min test, the high power output necessitates a very high cadence. As a subjects' ability to produce power declines, so too does cadence. In order to ensure that cadence does not fall to unacceptably low levels, the researcher must adjust the Lode's power/cadence settings. This is done by adjusting the 'linear factor' $\alpha$ in the equation Power $=\alpha^{*} \mathrm{RPM}^{2}$. To date, researchers have adjusted the linear factor such that preferred cadence is reached at GET $+0.5 *\left(\dot{\mathrm{VO}}_{2 \max }-\mathrm{GET}\right)$ (i.e., $\left.50 \% \Delta\right)$, where GET is the gas exchange threshold. Given that $50 \% \Delta$ is very close to $\mathrm{CP}(46.7 \% \Delta$ in Vanhatalo et al. [30]), it is possible that the use of a Lode ergometer biases the 3-min all-out test towards an End Power close to GET and therefore to CP.

Estimates for EP, CP1 and CP2 reported in the present study may have been influenced by the selection of subjects. While previous studies $[2,9,30]$ utilized a range of athlete abilities, the present study was conducted on a relatively homogeneous sample of trained cyclists. This suggests that subjects in the present study, who are accustomed to high-intensity cycling performances, may have been better able to sustain their 3-min effort to ensure that $W^{\prime}$ was not depleted.

Mean $W^{\prime} 1(18.3 \mathrm{~kJ})$ and mean $W^{\prime} 2(16.6 \mathrm{~kJ})$ were also higher than in the subject group investigated by Vanhatalo et al. [30] (16 kJ). It is possible that subjects with a higher $W^{\prime}$ take longer to fully expend $W^{\prime}$ than those with a smaller $W^{\prime}$ using the isokinetic mode, a mode in which resistance is modulated according to fatigue level while maintaining cadence. This might suggest the need for an all-out test longer than $3 \mathrm{~min}$. However, this does not appear to be supported by the power profile in the present study, in which power declined towards a relative plateau over a similar time course to that described by Vanhatalo et al. [30]. Bergstrom et al. [5] recently reported $150 \mathrm{~s}$ EP derived from a similar method as the 3-min test using a Lode ergometer and which did not significantly differ from EP observed in the original 180 s test duration.

While it is not clear whether or not $W^{\prime}$ describes a true 'anaerobic work capacity' [13], if valid, the 3-min test would nevertheless provide a valuable tool for the assessment of this parameter. However, the data reported in the present study suggest that the anaerobic parameters derived from the 3-min test significantly underestimate $W^{\prime}$. This supports Vanhatalo et al. [30] who reported a WEP markedly below $W^{\prime}$ in 6 of 10 subjects. Vanhatalo et al. [30] suggested that the discrepancy might be the result of different acceleration profiles of the flywheel during all-out and constant work rate exercise when using the Lode ergometer. The suggestion is supported by the results in the present study as the SRM ergometer uses flywheel technology similar to the Lode ergometer.

The generalization of the $\mathrm{CP}$ concept to all-out exercise is dependent upon the capacity of the all-out trial to fully deplete $W^{\prime}$. Despite satisfying the requirements of the 3-min test [25], it might be possible that the present subjects were unable to fully deplete $W^{\prime}$. This is surprising given that a maximal accumulated oxygen deficit has been demonstrated following 60-90s of allout exercise [18,33]. Such observations led Brickley et al. [8] and Dekerle et al. [15] to evaluate whether a 90-s all-out test could estimate CP in adults and children, respectively. As in the present study, testing was conducted on an SRM ergometer using the isokinetic mode, and EP was significantly higher than CP. Despite a plateau being apparent in the final 10 s of the 90 -s test, Dekerle et al. [15] suggested that power output continues to decline at the end of the test. This led to the hypothesis that a test of longer duration would allow CP to be attained [8]. The hypothesis is refuted by the observation that the results of the current investigation agree so closely with those obtained when using the 90-s test to derive CP.

Following the protocol proposed by Vanhatalo et al. [30] while using an isokinetic mode might explain different outcomes between $\mathrm{EP}$ and $\mathrm{CP} 1 / \mathrm{CP} 2$. To investigate the robustness of the 3-min all-out test, Vanhatalo et al. [31] manipulated the flywheel resistance for subjects to achieve EP cadences which were $\pm 10 \mathrm{rev} \cdot \mathrm{min}^{-1}$ different from the original investigation. The authors reported no differences in EP for reduced cadence 
values and a reduced EP when applying a higher cadence strategy. Consistent with the standard protocol, subjects in the present study applied their preferred cadence throughout testing but on average had a higher cadence $\left(95 \pm 8 \mathrm{rev} \cdot \mathrm{min}^{-1}\right)$ when compared to Vanhatalo et al. [29] $\left(88 \pm 6 \mathrm{rev} \cdot \mathrm{min}^{-1}\right)$. The standard all-out protocol [30] requires subjects to adopt their preferred cadence, but the standard test conditions can be sensitive to minor variations in the ergometer resistance settings. Carnevale and Gaesser [11] as well as Barker et al. [3] investigated the impact of pedalling speed on the power-duration relationship. Both studies reported a lower $\mathrm{CP}$ and an unaffected $W^{\prime}$ when employing a high $\left(100 \mathrm{rev} \cdot \mathrm{min}^{-1}\right)$ vs. a low $\left(60 \mathrm{rev} \cdot \mathrm{min}^{-1}\right)$ cadence strategy. The differences in cadence between the present study and Vanhatalo et al. [30] could be partly responsible for the observed discrepancies between EP and CP1/CP2.

\section{Conclusion}

$\nabla$

The findings of the present study suggest that it may not be possible to generalize the $\mathrm{CP}$ concept for use on all ergometer models or modes. The 'aerobic' (EP) and 'anaerobic' $\left(W^{\prime}\right)$ parameters derived from 3-min all-out cycle test are significantly different to the 'aerobic' and 'anaerobic' parameters derived from the standard work-time and power $^{-1} /$ time $^{\mathrm{CP}}$ model. Using only cyclists with a preferred cadence $\geq 90 \mathrm{rev} \cdot \mathrm{min}^{-1}$, or validation studies using rowing, self-powered treadmill ergometers, or track running or cycling might shed some further light on the different outcomes of our study.

\section{Acknowledgements}

The authors gratefully acknowledge the cooperation of the athletes who took part in this study. The authors would also like to thank Dr. Enid Ginn for her assistance during the preparation of Experiment.

\section{References}

1 Atkinson G, Nevill AM. Statistical methods for assessing measurement error (reliability) in variables relevant to sports medicine. Sports Med 1998; 26: 217-238

2 Barker AR, Bond B, Toman C, Williams CA, Armstrong N. Critical power in adolescents: physiological bases and assessment using all-out exercise. Eur J Appl Physiol 2012; 112: 1359-1370

3 Barker T, Poole DC, Noble ML, Barstow TJ. Human critical power-oxygen uptake relationship at different pedalling frequencies. Exp Physiol 2006; 91: 621-632

4 Bergstrom H, Housh T. A new single work bout test to estimate critical power and anaerobic work capacity. J Strength Cond Res 2012; 26: 656-663

5 Bergstrom H, Housh TJ, Zuniga JM, Camic CL, Traylor DA, Lewis RW, Schmidt RJ, Johnson GO. Estimates of critical power and anaerobic work capacity from a single, all-out test of less than 3-min. J Sport Med Doping Stud 2012; 2: 1-5

6 Blair SN, Painter P, Pate R, Pate R, Smith K, Taylor C. Resource manual for Guidelines for exercise testing and prescription. Philadelphia: Lea \& Febiger, 1988; 414-420

7 Bland JM, Altman DG. Statistical methods for assessing agreement between two methods of clinical measurement. Lancet 1986; 1 : $307-310$
8 Brickley G, Dekerle J, Hammond AJ, Pringle J, Carter H. Assessment of maximal aerobic power and critical power in a single 90 -s isokinetic all-out cycling test. Int J Sports Med 2007; 28: 414-419

9 Burnley M, Doust JH, Vanhatalo A. A 3-min all-out test to determine peak oxygen uptake and the maximal steady state. Med Sci Sports Exerc 2006; 38: 1995-2003

10 Busso T, Gimenez P, Chatagnon M. A comparison of modelling procedures used to estimate the power-exhaustion time relationship. Eur J Appl Physiol 2010; 108: 257-263

11 Carnevale TJ, Gaesser GA. Effects of pedaling speed on the powerduration relationship for high-intensity exercise. Med Sci Sports Exerc 1991; 23: 242-246

12 Cheng CF, Yang YS, Lin HM, Lee CL, Wang CY. Determination of critical power in trained rowers using a three-minute all-out rowing test. Eur J Appl Physiol 2012; 112: 1251-1260

13 Dekerle J, Brickley G, Hammond AJP, Pringle JSM, Carter H. Validity of the two-parameter model in estimating the anaerobic work capacity. Eur J Appl Physiol 2006; 96: 257-264

14 Dekerle J, Vanhatalo A, Burnley M. Determination of critical power from a single test. Sci Sports 2008; 23: 231-238

15 Dekerle J, Williams C, McGawley K, Carter H. Critical power is not attained at the end of an isokinetic 90-second all-out test in children. J Sports Sci 2009; 27: 379-385

16 Francis JT, Quinn TJ, Amann M, LaRoche DP. Defining intensity domains from the end power of a 3-min all-out cycling test. Med Sci Sports Exerc 2010; 42: 1769-1775

17 Gaesser GA, Wilson LA. Effects of continuous and interval training on the parameters of the power-endurance time relationship for highintensity exercise. Int J Sports Med 1988; 9: 417-421

18 Gastin PB, Costill DL, Lawson DL, Krzeminski K, McConell GK. Accumulated oxygen deficit during supramaximal all-out and constant intensity exercise. Med Sci Sports Exerc 1995; 27: 255-263

19 Gastin PB, Lawson DL. Variable resistance all-out test to generate accumulated oxygen deficit and predict anaerobic capacity. Eur J Appl Physiol 1994; 69: 331-336

20 Harriss DJ, Atkinson G. Update - Ethical standards in sport and exercise science research. Int J Sports Med 2011; 32: 819-821

21 Hill $D$, Poole DCSJ. The relationship between power and the time to achieve VO2max. Med Sci Sports Exerc 2002; 34: 709-714

22 Hill DW. The critical power concept. A review. Sports Med 1993; 16: 237-254

23 Hopkins WG. A new view on statistics. Internet Society for Sport Science 2000. Available at: http://www.sportsci.org/resource/stats/ Accessed 15 November 2012

24 Johnson TM, Sexton PJ, Placek AM, Murray SR, Pettitt RW. Reliability analysis of the 3-min all-out exercise test for cycle ergometry. Med Sci Sports Exerc 2011; 43: 2375-2380

25 Jones AM, Vanhatalo A, Burnley M, Morton RH, Poole DC. Critical power: implications for determination of VO2max and exercise tolerance. Med Sci Sports Exerc 2010; 42: 1876-1890

26 Parker Simpson L, Jones AM, Vanhatalo A, Wilkerson DP. Influence of initial metabolic rate on the power-duration relationship for all-out exercise. Eur J Appl Physiol 2012; 112: 2467-2473

27 Passfield L, Jobson SA, Atkinson G, Barton G, Scarf P. Longitudinal changes in weighted indices of work-rate but not average power output of cyclists during a racing season [abstract]. J Sports Sci 2009; 27: S102-S103

28 Paton C, Hopkins W. Variation in performance of elite cyclists from race to race. Eur J Sport Sci 2006; 6: 25-31

29 Poole DC. Resolving the determinants of high-intensity exercise performance. Exp Physiol 2009; 94: 197-198

30 Vanhatalo A, Doust JH, Burnley M. Determination of critical power using a 3-min all-out cycling test. Med Sci Sports Exerc 2007; 39: 548-555

31 Vanhatalo A, Doust JH, Burnley M. Robustness of a 3 min all-out cycling test to manipulations of power profile and cadence in humans. Exp Physiol 2008; 93: 383-390

32 Vanhatalo A, Jones AM. Influence of creatine supplementation on the parameters of the "all-out critical power test". J Exerc Sci Fit 2009; 7: 9-17

33 Withers RT, Ploeg G, Finn JP. Oxygen deficits incurred during 45, 60, 75 and 90-s maximal cycling on an air-braked ergometer. Eur J Appl Physiol 1993; 67: 185-191 